\title{
Michel Graulich (1944-2015)
}

\section{Guilhem Olivier}

\section{OpenEdition}

\section{Journals}

Édition électronique

URL : https://journals.openedition.org/jsa/14337

DOI : $10.4000 /$ jsa. 14337

ISSN : 1957-7842

\section{Éditeur}

Société des américanistes

\section{Édition imprimée}

Date de publication : 31 décembre 2015

Pagination : 297-306

ISSN : 0037-9174

\section{Référence électronique}

Guilhem Olivier, « Michel Graulich (1944-2015) », Journal de la Société des américanistes [En ligne],

101-1 et 2 | 2015, mis en ligne le 15 mars 2016, consulté le 24 février 2023. URL : http://

journals.openedition.org/jsa/14337 ; DOI : https://doi.org/10.4000/jsa.14337 
NÉCROLOGIE 



\section{Michel Graulich (1944-2015)}

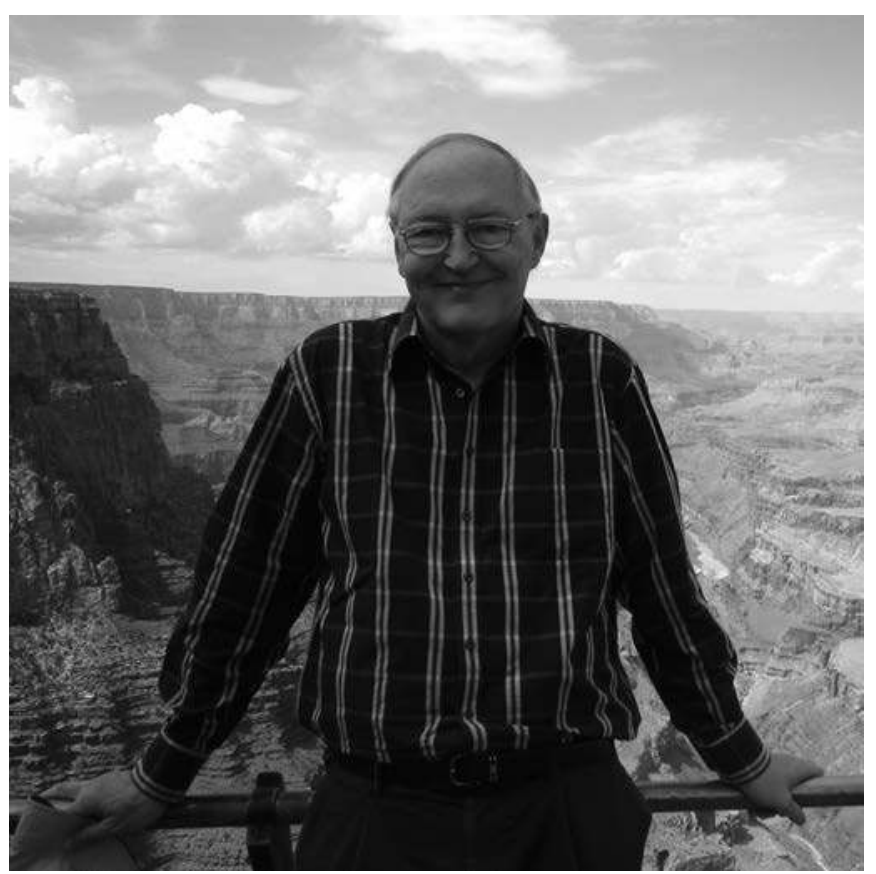

Michel Graulich au Grand Canyon en 2011.

C'est avec une immense tristesse que nous avons appris la mort de Michel Graulich, le 11 février 2015 à Bruxelles.

Né en 1944 à Orroir (Belgique), Graulich suit des études d'histoire de l'antiquité à l'université de Gand, puis d'histoire de l'art et d'archéologie à l'université libre de Bruxelles, où il obtient un doctorat en 1980, intitulé « Mythes et rituels du Mexique ancien préhispanique », préparé sous la direction d'Annie Dorsinfang-Smets. Lui-même professeur à l'université libre de Bruxelles, il fut également directeur d'études à l'École pratique des hautes études, section des sciences religieuses.

L'originalité et la solidité de son œuvre se manifestent pleinement dès la publication de sa thèse de doctorat: Mythes et rituels du Mexique ancien préhispanique (1987). Pour la première fois, un chercheur propose une interprétation globale à la fois des mythes et des rituels de l'ancien Mexique central. Inspiré par les travaux de Georges Dumézil et de Claude Lévi-Strauss, Graulich entreprend de reconstruire à partir de sources fragmentaires la mythologie 
mésoaméricaine, n'hésitant pas à utiliser des mythes mayas - comme ceux inclus dans le Popol Vuh - et des mythes indigènes actuels à des fins comparatives. Parmi les principaux apports de cette reconstruction, signalons l'importance du mythe de Tamoanchan, un lieu paradisiaque d'où les dieux sont chassés suite à une transgression. L'approche comparative suivie par Graulich lui permet de réfuter au passage l'idée d'une prétendue influence chrétienne sur les mythes de paradis perdus, puisque, en réalité, ces derniers sont largement répandus dans le monde. Quoi qu'il en soit, il démontre que le mythe d'origine du quatrième soleil à Teotihuacan - où les dieux sont sacrifiés, deux d'entre eux se transformant en Soleil et Lune - explique comment les divinités parviennent à vaincre l'obscurité et la mort, regagnent l'empyrée d'où ils avaient été exilés et créent les au-delàs - le Soleil et le Tlalocan - où iront les méritants. De là, l'importance de l'acte sacrificiel qui constitue à la fois un acte de purification et un moyen d'alléger le corps pour vaincre la mort, acte sacrificiel des dieux que les hommes se doivent de reproduire sur la terre. Quant au mythe de la guerre sacrée, dont les victimes alimenteront le Soleil et la Terre, il rend compte du caractère double du sacrifice humain, dédié à la fois à l'astre du jour et à la Terre.

Graulich analyse ensuite le rôle fondamental assigné aux cycles cosmiques (les fameux « Soleils » ou « ères ») dans la pensée mésoaméricaine. Les récits les concernant manifestent l'importance des concepts d'équilibre, de rupture et d'inversion, chacune des ères étant placée sous la tutelle d'une divinité - Quetzalcoatl et Tezcatlipoca - qui alternent comme « Soleil». Cette approche originale lui permet d'expliquer certaines « contradictions » des sources quant à l'ordre des Soleils. En effet, les Mexicas introduisirent un cinquième Soleil, celui de leur divinité tutélaire Huitzilopochtli, et ils permutèrent le Soleil d'Eau et le Soleil de Terre. C'est également à partir de ce modèle des différents Soleils que Graulich peut analyser de manière brillante les récits de 1' " histoire » toltèque. Dans le cadre du quatrième Soleil, Quetzalcoatl apparaît successivement comme Soleil conquérant, qui vainc et sacrifie les meurtriers de son père Mixcoatl, puis comme astre lunaire au déclin à Tollan - cité paradisiaque assimilée au Tlalocan -, et enfin comme Vénus après sa crémation sur un bûcher qui annonce sa renaissance. Dans Quetzalcóatl y el espejismo de Tollan (1988), Graulich reprend et approfondit l'étude de ce personnage central de la mythologie mésoaméricaine, en citant systématiquement les sources sur lesquelles se fondent ses interprétations.

Mais revenons à la deuxième partie de Mythes et rituels..., où sont analysées les fêtes des vingtaines à l'aune d'une nouvelle interprétation du calendrier rituel. D'après Graulich, en l'absence de bissexte, les Mexicas - comme d'ailleurs les Mayas - auraient de fait volontairement laissé leur calendrier « solaire » se décaler afin de ne pas affecter sa concordance avec le calendrier de 260 jours et avec les cycles vénusiens. À partir des noms des vingtaines, dont certains renvoient à des époques précises de l'année (début ou fin de la saison des pluies 
par exemple), il aboutit à une remise en place des vingtaines telles qu'elles auraient été situées au moment de leur « position originelle », vers 680-683. Cette reconstruction débouche sur une nouvelle analyse des fêtes des vingtaines, lesquelles, quoique décalées par rapport à l'année solaire, auraient effectivement eu une signification saisonnière. Les résultats détaillés de cette lecture originale sont consignés dans Ritos aztecas. Las fiestas de las veintenas (1999).

Graulich s'est intéressé ensuite à une figure particulièrement importante de l'histoire aztèque : Montezuma Xocoyotzin ou Motecuhzoma II, auquel il a consacré l'ouvrage intitulé Montezuma ou l'apogée et la chute de l'empire aztèque (1994). À l'instar de Jacques Le Goff qui, dans son grand livre sur Saint Louis (Gallimard, Paris, 1996), se consacre à la difficile recherche de la personnalité de Louis IX - au-delà du stéréotype du roi et du modèle du saint -, Graulich se demande dans quelle mesure un portrait fidèle d'un personnage préhispanique est réalisable à partir de sources souvent partisanes, voire contradictoires. De la même façon que Le Goff présente, dans sa biographie, la société, la religion, l'art et l'imaginaire de la France du XIII ${ }^{\mathrm{e}}$ siècle, Graulich dresse, dans son propre livre, un tableau détaillé du Mexique du début du XVI ${ }^{\mathrm{e}}$ siècle, dépeignant la structure de l'Empire aztèque, la vie quotidienne de ses habitants et les mythes qui constituent les bases de leur « vision du monde ». En fait, la mobilisation des mythes pour analyser les actes de Motecuhzoma II constitue un des aspects les plus stimulants de l'ouvrage.

Pour l'étude de la vie du dernier souverain mexica, nous disposons principalement des récits des Espagnols, certains témoins directs des événements de la Conquête, et des écrits des Indiens et des religieux qui réalisèrent leurs œuvres à l'époque coloniale. Confrontant ces sources entre elles, Graulich conclut que les premières, en général, sont plus fiables que les écrits qui forment ce que l'on a appelé « la vision des vaincus ». Cela étant, la « confiance » de Graulich vis-à-vis des sources espagnoles est relative ; il démontre, par exemple, que l'Historia verdadera de la conquista... de Bernal Díaz del Castillo doit être employée avec circonspection. Quant aux sources indigènes, elles furent élaborées après la Conquête, souvent pour minimiser la résistance aux envahisseurs voire pour exalter la collaboration de certains groupes avec les Espagnols. Cela étant, les sources indigènes s'avèrent fondamentales pour appréhender les schémas mythiques préhispaniques qui présidèrent aux descriptions autochtones de la Conquête tout en expliquant certaines actions de Motecuhzoma II.

Il vaut la peine de s'attarder ici sur quelques points particulièrement importants de la première partie du livre, dédiée à la période qui précède l'arrivée des Espagnols. Graulich démontre que les campagnes militaires incessantes durant le règne de Motecuhzoma II visaient à consolider l'empire en y intégrant des enclaves indépendantes, et surtout à saper la puissance de la vallée de Puebla, où résidaient les principaux adversaires de la Triple Alliance. De la sorte, il explique que la célèbre « guerre fleurie » avait pour but, outre la capture de 
futurs sacrifiés, de contrôler et d'isoler les ennemis traditionnels de la Triple Alliance. Sur le plan religieux, Motecuhzoma II promut des changements significatifs, comme le passage de la fête du Feu nouveau de l'année 1 Lapin à une année 2 Roseau. En effet, 2 Roseau était le nom calendaire de Tezcatlipoca qui, identifié avec Huitzilopochtli, patronnait le cinquième Soleil des Mexicas. Cette « véritable révolution religieuse " devait entraîner le passage de la fête du Feu nouveau de la vingtaine d'ochpaniztli à celle de panquetzaliztli dont la signification allait changer. Alors que cette dernière vingtaine commémorait le sacrifice de Nanahuatl-Quetzalcoatl qui se transforma en Soleil, avec la réforme de Motecuhzoma II la fête de panquetzaliztli en vint à reproduire la victoire de Huitzilopochtli, Soleil des Mexicas, sur les forces nocturnes. Cette substitution de Quetzalcoatl par Huitzilopochtli aura pourtant de graves conséquences lorsque les Espagnols furent assimilés au « Serpent à plumes » qui revenait pour « récupérer son royaume ».

L'un des éléments les plus novateurs de l'ouvrage de Graulich consiste à démystifier la prétendue lâcheté de Motecuhzoma II face aux Espagnols. Plusieurs témoignages laissent penser que le roi mexica jugeait son empire riche mais décadent, sur le point de disparaître, à l'image de l'Empire toltèque qui l'avait précédé. Toutefois, après avoir pris la mesure des envahisseurs, Motecuhzoma II adopta une attitude volontaire pour contrecarrer la progression des troupes espagnoles vers sa capitale. Graulich révèle que le souverain mexica s'inspira, à l'occasion, de précédents mythiques. Cela explique un épisode énigmatique qui eut lieu alors que les Espagnols se trouvaient encore sur la côte du Golfe du Mexique. Motecuhzoma II envoya à leur rencontre un dignitaire qui ressemblait tant au capitaine des conquistadors que les Espagnols eux-mêmes, amusés, l'appelèrent Cortez ! Graulich détecte avec finesse que le tlatoani cherchait à reproduire de cette façon la manœuvre de Tezcatlipoca à Tollan qui avait présenté un miroir face à Quetzalcoatl afin de l'affaiblir et de le tromper.

Parallèlement, Motecuhzoma II employa des moyens que nous jugerions aujourd'hui plus « concrets » pour lutter contre les envahisseurs. Prenons l'exemple du massacre de Cholula, fréquemment cité pour condamner la violence gratuite de Cortez et de ses troupes. D’après Graulich, plusieurs éléments confirment la thèse d'une embuscade : accusé par Cortez, Motecuhzoma II avoua que certaines de ses troupes se trouvaient dans les environs de la ville. Argument capital, la garnison espagnole de Veracruz fut attaquée précisément au même moment qu'avait lieu l'embuscade à Cholula. C'est pourquoi l'image du tlatoani orgueilleux et tyrannique, responsable de la défaite, doit être placée parmi les mythes forgés par les sources indigènes, selon des précédents mythiques où des personnages transgresseurs entraînent la chute de leurs empires. S'inscrivant en faux contre l'image d'Indiens sans défense, paralysés par de superstitieuses angoisses et qui ne réagirent pour se défendre qu'acculés par les sanguinaires envahisseurs, Graulich 
démontre brillamment que les Mexicas - peuple guerrier et conquérant, faut-il le rappeler ? - résistèrent et, surtout, que Motecuhzoma II ne fut pas ce souverain superstitieux et lâche qui aurait trahi son peuple sans combattre.

L'intérêt de Graulich pour le thème du sacrifice humain remonte à ses premières années de recherches sur le Mexique ancien puisqu'il y consacra son mémoire de licence. Suite à une série de cours à l'EPHE, il publia, en 2005, Le sacrifice humain chez les Aztèques, véritable somme sur ce sujet, à la fois polémique et passionnant. Inutile d'insister sur l'érudition et le caractère systématique de la recherche qu'il effectua alors dans toutes les sources disponibles (archéologiques, pictographiques et écrites), non seulement sur les Mexicas mais aussi sur d'autres peuples de l'ancien Mexique. Quant à son approche comparatiste - déjà employée dans ses précédents ouvrages -, elle prend ici une dimension plus ample encore. En témoigne le premier chapitre dédié aux mythes, où il n'hésite pas à comparer certains motifs mythiques mésoaméricains (comme l'arbre interdit du paradis) avec des récits de la Bible, de l'Inde ancienne ou encore de peuples actuels d'Afrique ou d'Australie. Graulich applique d'ailleurs parfois cette approche comparatiste aux rituels : ainsi, lorsqu'il évoque les Khonds de l'Inde qui faisaient pleurer les victimes sacrificielles afin de susciter des pluies abondantes, tout comme les Mexicas qui associaient les sanglots des enfants destinés au sacrifice et les futures précipitations. De même, à propos du mythe de Yappan - un personnage qui, à travers des pratiques ascétiques, menace de se transformer en scorpion à la piqûre mortelle - Graulich a recours à des données de l'Inde où les dieux sont parfois menacés par des ascètes dont les pénitences augmentent la puissance. Sans succomber à la tentation diffusionniste ou à des simplifications abusives, sa « méthode comparatiste » prudente et rigoureuse ouvre incontestablement de nouvelles perspectives interprétatives, au-delà d'une singularité mésoaméricaine qui parfois s'apparente à un isolationnisme obtus.

Au début de l'ouvrage, Graulich présente une brève historiographie du sacrifice humain en Mésoamérique. Pour simplifier, nous dirons que depuis le $\mathrm{XIX}^{\mathrm{e}}$ siècle, deux grands types d'interprétation ont été avancés : la première, " énergétique », où on considère le sacrifice comme un moyen d'alimenter et de vivifier les divinités et ce qu'elles représentent; la seconde, plus « spirituelle », et selon laquelle l'acte sacrificiel rachète les fautes et facilite l'accès à des au-delàs glorieux. Tout au long de l'ouvrage Graulich combine ces deux théories, les fonctions et les significations du sacrifice humain se révélant extrêmement diverses selon les participants et les contextes rituels. C'est ainsi qu'il développe l'hypothèse selon laquelle le sacrifice représentait un châtiment et une expiation. En effet, la présence des dieux exilés sur la terre au moment de la création du Soleil et de la Lune à Teotihuacan ne s'explique que par la transgression dont ils furent les acteurs au « paradis » de Tamoanchan. Suite à leur sacrifice, les dieux regagnèrent le ciel où furent créés les au-delàs du Soleil 
et de la Lune, destinées post-mortem des deux grandes catégories de sacrifiés : les guerriers, accompagnateurs de l'astre solaire, et les esclaves rituellement baignés, destinés à atteindre le Tlalocan, situé dans la lune. Transgresseurs également - ils n'offrent pas leur gibier à Soleil et Terre, s'enivrent et couchent avec des femmes -, les Mimixcoa apparaissent dans le mythe d'origine de la guerre sacrée comme les prototypes des guerriers sacrifiés.

La deuxième partie de l'ouvrage est consacrée aux différents acteurs et au déroulement du sacrifice. Graulich insiste sur le rôle fondamental des sacrifiants - reprenant la définition classique d'Hubert et de Mauss - qui s'identifient à leurs victimes, meurent symboliquement par leur truchement et ainsi acquièrent des mérites et la promesse d'un au-delà glorieux. Il détecte dans les sources les preuves de cette identification entre les sacrifiants et leurs victimes, par exemple dans le fait que les guerriers étaient ornés à l'image des victimes sacrificielles qu'ils avaient capturées, victimes identifiées aux Mimixcoa. Graulich remarque aussi que les prisonniers de guerre étaient dans un premier temps intégrés à la communauté et, comme chez les Tupinambas du Brésil, on leur offrait parfois des épouses rituelles. Quant aux esclaves obtenus par les campagnes militaires, le tribut des cités conquises, ou faits tels par les tribunaux, ils représentaient la deuxième catégorie de sacrifiés. Certains étaient baignés rituellement et personnifiaient les dieux durant les rites. Étant donné l'absence ici de cérémonie d'intégration au groupe, Graulich considère que ces esclaves appartenaient de facto à la communauté.

Graulich ne néglige pas la dimension sociale du sacrifice humain et il souligne l'aspect public des rites durant lesquels le sacrifiant était exposé à la vue de divers groupes sociaux avec lesquels il établissait des relations de réciprocité à travers des dons. Ainsi, il explique : « le sacrifiant recueille ici-bas gloire, prestige, promotion et relations. Son goût de la guerre et son esprit guerrier sont encouragés, le butin, les dons et les récompenses l'enrichissent, l'intérêt de l'État est servi » (p. 164). Le dernier chapitre de l'ouvrage est, lui, consacré au déroulement du sacrifice. Graulich y examine avec minutie les différents espaces où avaient lieu les sacrifices (temples, autels, pierres de sacrifice, lieux d'exposition des crânes) ainsi que les instruments du rite. Les différentes techniques d'exécution font également l'objet de descriptions précises - extraction du cœur, décapitation, crémation, sacrifice à coups de flèches - à partir des sources anciennes et des travaux des anthropologues physiques.

La dernière partie de l'étude est consacrée au « festin cannibale », un thème éminemment débattu. Outre les incontestables aspects rituels du cannibalisme, Graulich souligne ses composantes vindicatives et gastronomiques, en particulier vis-à-vis des guerriers capturés sur le champ de bataille. Il examine l'importance de la « cuisine du sacrifice aztèque », les victimes bouillies relevant de la culture (sacrifice religieux), tandis que celles rôties, pratique associée à la nature, renverraient à un sacrifice de type alimentaire. 
En conclusion, Graulich aborde les possibles explications de la multiplication des sacrifices humains dans l'ancien Mexique : absence d'animaux domestiques de grande taille qui constituèrent, dans d'autres civilisations, les victimes privilégiées ; raisons sociales inspirées du modèle de René Girard, la participation/complicité aux sacrifices consolidant une cohésion sociale fragile dans de grandes cités pluriethniques.

L'espace manque pour rendre compte de la totalité de l'œuvre de Graulich. Outre les sujets abordés dans ses principaux ouvrages, il a publié aussi de nombreux articles dans lesquels il précise certains points de ses démonstrations, parfois dans le cadre de discussions avec des collègues. On ne saurait oublier, d'autre part, qu'il a consacré plusieurs écrits importants à l'art de la Mésoamérique et, en particulier, aux principaux monuments religieux aztèques : du Teocalli de la Guerre sacrée à la Coatlicue, en passant par le fameux Calendrier aztèque ou le Temalacatl-Cuauhxicalli d'Axayacatl, les analyses iconographiques de Graulich allient la précision des descriptions, une érudition sans faille et l'originalité des interprétations.

Ce qui précède a tenté de montrer dans quelle mesure la disparition prématurée de Michel Graulich laisse dans le milieu des américanistes un vide immense. Tous ceux qui ont eu le privilège de connaître Michel, étudiants, collègues et amis, garderont le souvenir d'un maître rigoureux et généreux à la fois, doté d'une immense culture alliée à une grande sensibilité et à un sens de l'humour hors du commun.

Guilhem OLIVIER

Instituto de Investigaciones Históricas, UnAm, Mexico.

\section{Publications, une sélection}

\section{Livres}

1987, Mythes et rituels du Mexique ancien préhispanique, Académie royale de Belgique, Bruxelles.

1988, Quetzalcóatl y el espejismo de Tollan, Instituut voor Amerikanistiek, Antwerpen. 1994, Montezuma ou l'apogée et la chute de l'empire aztèque, Fayard, Paris.

1995, Codex Azcatitlan, introd. de Michel Graulich, comment. de Robert H. Barlow, mis à jour par Michel Graulich, trad. espagnole Leonardo López Luján, trad. française Dominique Michelet, Bibliothèque nationale de France/Société des américanistes, Paris.

1999, Ritos aztecas: las fiestas de las veintenas, Instituto Nacional Indigenista, Mexico. 2005, Le sacrifice humain chez les Aztèques, Fayard, Paris. 


\section{Articles}

1974, «Las peregrinaciones aztecas y el ciclo de Mixcóatl », Estudios de cultura náhuatl, 11, p. 311-354.

1981, « The metaphor of the day in Ancient Mexican myth and ritual », Current anthropology, 22 (1), p. 45-60.

1982, «Les mises à mort doubles dans les rites sacrificiels des anciens Mexicains ", Journal de la société des américanistes, 68, p. 49-58.

1983, « Myths of paradise lost in Pre-Hispanic Central Mexico », Current anthropology, 24 (5), p. 575-588.

1983, « Vues obliques et troisième dimension dans l'art maya », Baessler-Archiv, 31, p. 185-218.

1984, «Quelques observations sur les sculptures mésoaméricaines dites “ChacMool” », Jaarboek van het Vlaams Instituut voor Amerikanistiek, 1984, p. 51-72.

1987, «Les incertitudes du Grand Temple », in Arne Eggebrecht (éd.), Les Aztèques. Trésors du Mexique ancien, catalogue, Roemer-Museum/Pelizaeus-Museum, Hildesheim, p. 121-131, 224-227.

1990, « Dualities in Cacaxtla », in Rudolph van Zantwijk, Rob de Ridder et Edwin Braakhuis (éd.), Mesoamerican dualism (46th International congress of Americanists, Amsterdam 1988), RUU-ISOR, Utrecht, p. 94-118.

1990, «La royauté inca », in Inca-Perú. 3000 ans d'histoire, catalogue d'exposition (Musées royaux d'art et d'histoire, Bruxelles, 21 septembre-30 décembre 1990), Imschoot, Gent, vol. 1, p. 426-437.

1991, «Les grandes statues aztèques dites de Coatlicue et de Yollotlicue », in Raquel Thiercelin (éd.), Cultures et société. Andes et Méso-Amérique. Mélanges en hommage à Pierre Duviols, Publication de l'université de Provence, Aix-enProvence, vol. 1, p. 375-419.

1991, « L'inauguration du temple principal de Mexico en 1487 », Revista española de antropología americana, 21, p. 121-143.

1992, "Quetzalcoatl-Ehecatl. The bringer of life », in Nicolas Saunders (éd.), Ancient America. Contributions to New World archaeology, Oxbow Books, Oxford, p. 33-38.

1992, "Les fêtes mobiles des Aztèques », Annuaire de l'École pratique des hautes études. Section des sciences religieuses, 99, p. 31-37.

1993, «Les Aztèques avaient-ils une religion? », in Ugo Bianchi (éd.), The notion of « religion » in comparative research. Selected proceedings of the XVI International association of history of religions congress, L'Erma, Roma, p. 239-245.

1995, « Bonampak: la lógica de las pinturas », in Carmen Varela Torrecilla, Juan Luis Bonor Villarejo et María Yolanda Fernández Marquínez (éd.), Religión y sociedad en el área maya, Sociedad Española de Estudios Mayas (Publicación de la Sociedad Española de Estudios Mayas, 3), Madrid, p. 43-50.

1995, «Una posible explicación del punto de partida de la cuenta larga maya », in Carmen Varela Torrecilla, Juan Luis Bonor Villarejo et María Yolanda Fernández Marquínez (éd.), Religión y sociedad en el área maya, Sociedad Española de Estudios Mayas (Publicación de la Sociedad Española de Estudios Mayas, 3), Madrid, p. 51-56. 
1995, « El Popol Vuh en el Altiplano mexicano », in Memorias del segundo Congreso internacional de mayistas, Universidad Nacional Autónoma de México, Instituto de Investigaciones Filológicas, Centro de Estudios Mayas, Mexico, p. 117-130.

1995, « Fêtes mobiles et occasionnelles des Aztèques (suite) », Annuaire de l'École pratique des hautes études. Section des sciences religieuses, 102, p. 25-31.

1996, "Fêtes mobiles et occasionnelles des Aztèques (suite et fin) », Annuaire de l'École pratique des hautes études. Section des sciences religieuses, 103, p. 33-39.

1996, « Jésus, Horus, Shiva et Quetzalcoatl. De quelques similitudes entre les mythes de l'Ancien et du Nouveau Monde ", Académie royale des sciences d'outre-mer. Bulletin des séances, 42 (3), p. 397-410.

1996, “"La mera verdad resiste a mi rudeza": forgeries et mensonges dans l'Historia verdadera de la conquista de la Nueva España de Bernal Díaz del Castillo », Journal de la société des américanistes, 82, p. 63-95.

1997, « Reflexiones sobre dos obras maestras del arte azteca: la Piedra del Calendario y el Teocalli de la Guerra Sagrada », in Xavier Noguez et Alfredo López Austin (éd.), De hombres y dioses, El Colegio de Michoacán, Michoacán/El Colegio Mexiquense, Zinacantepec, p. 155-207.

1997, «Elementos de las fiestas de las veintenas en las trecenas del Códice Borbónico », in Salvador Rueda Smithers, Constanza Vega Soza et Rodrigo Martínez Baracs (éd.), Códices y documentos sobre México. Segundo simposio, Instituto Nacional de Antropología e Historia, Mexico, p. 205-20.

1997, "Chasse et sacrifice humain chez les Aztèques », Académie royale des sciences d'outre-mer. Bulletin des séances, 43 (4), p. 433-446.

1998, «La royauté sacrée chez les Aztèques de Mexico », Estudios de cultura náhuatl, 28, p. 197-217.

1999, « Nota sobre el llamado "Cuauhxicalli-temalacatl de Motecuhzoma Ilhuicamina" », Cuicuilco, 5 (4), p. 239-250.

2000, "Les contenus subliminaux de l'image chez Felipe Guaman Poma de Ayala », Journal de la société des américanistes, 86, p. 67-112.

2000, « Tlahuicole, un héroe tlaxcalteca controvertido », in Federico Navarrete et Guilhem Olivier (éd.), El héroe entre el mito y la historia, Universidad Nacional Autónoma de México, Instituto de Investigaciones Históricas/Centro d'études mexicaines et centraméricaines, Mexico, p. 89-99.

2000, «Aztec human sacrifice as expiation », History of religions, 39 (4), p. 352-371.

2000, « Más sobre la Coyolxauhqui y las mujeres desnudas de Tlatelolco », Estudios de cultura náhuatl, 31, p. 77-94.

2002, « Los reyes de Tollan », Revista española de antropología americana, 32, p. $87-114$.

2002, « Tezcatlipoca-Omácatl, el comensal imprevisible», Cuicuilco, 9 (25), p. 359-367.

2002, "Acerca del "Problema de ajustes del año calendárico mesoamericano al año trópico" », Estudios de cultura náhuatl, 33, p. 45-56.

2004, « Relieves mexicas de Tlaltéotl-Tláloc con influencia teotihuacana », in Patrick Lesbre et Marie-José Vabre (éd.), Le Mexique préhispanique et colonial. Hommage à Jacqueline de Durand-Forest, L'Harmattan, Paris, p. 49-72. 
2005, «The warriors of the Bilimek Pulque Vessel », in Peter Eeckhout et Geneviève Le Fort (éd.), Wars and conflicts in Prehispanic Mesoamerica and the Andes, John and Erica Hedges/Archaeopress (BAR International Series, 1385), Oxford, p. 58-65.

2005, « Autosacrifice in Ancient Mexico », Estudios de cultura náhuatl, 36, p. 301-329. 2005, « Un relieve de Coyolxauhqui en Tetzcoco », Mexicon, 27 (1), p. 6-10.

2006, " Le codex aztèque du Palais Bourbon ou Codex Borbonicus ", Annuaire de l'École pratique des hautes études. Section des sciences religieuses, 113, p. 45-51.

2007, « Le codex aztèque du Palais Bourbon ou Codex Borbonicus (suite) », Annuaire de l'École pratique des hautes études. Section des sciences religieuses, 114, p. 41-47.

2008, « Nuevas consideraciones en torno al Teocalli de la Guerra Sagrada », in Guilhem Olivier (éd.), Símbolos de poder en Mesoamérica, Universidad Nacional Autónoma de México, Instituto de Investigaciones Históricas, Instituto de Investigaciones Antropológicas, Mexico, p. 163-174.

2008, « Las fiestas del año solar en el Códice Borbónico », Itinerarios, 8, p. 185-194.

\section{Articles en collaboration}

1978, «Une grande statue en terre cuite du Veracruz (Mexique) », Bulletin des Musées royaux d'art et d'histoire, 48, p. 35-48 (avec Michèle Doppée).

1993, « Decapitation among the Aztecs: mythology, agriculture and politics, and hunting », Estudios de cultura náhuatl, 23, p. 163-178 (avec Elizabeth Baquedano).

2004, « ¿Deidades insaciables? La comida de los dioses en el México antiguo », Estudios de cultura náhuatl, 35, p. 121-155 (avec Guilhem Olivier). 